\title{
Studies of the Magnetic Axis Switching in Magnetite
}

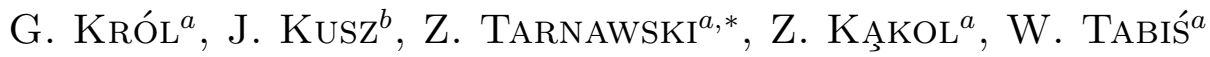 \\ AND A. KOZŁOWSKI ${ }^{a}$ \\ ${ }^{a}$ Department of Solid State Physics, Faculty of Physics and Applied Computer \\ Science, AGH University of Science and Technology \\ al. A. Mickiewicza 30, 30-059 Kraków, Poland \\ ${ }^{b}$ Institute of Physics, University of Silesia \\ Uniwersytecka 4, 40-007 Katowice, Poland
}

The influence of the external magnetic field on magnetic and crystallographic structure of magnetite was studied. We have confirmed, by means of vibrating sample magnetometer measurements, that external magnetic field can switch the easy magnetic axis to the new crystallographic direction. We have also proved by the direct observation of the crystalline structure that this phenomenon is strictly related to the crystallographic structure relaxation.

PACS numbers: $71.30 .+\mathrm{h}, 61.50 . \mathrm{Ks}, 75.30 . \mathrm{Gw}$

\section{Introduction}

In magnetite below the Verwey transition at $T_{\mathrm{V}} \approx 124 \mathrm{~K}$ some cation ordering develops that affects many physical properties. Since the orthodox meaning of charge ordering concept developed by Verwey is now questioned [1-3], the problem of the Verwey transition is again open for the discussion. Here we present the introductory experiments intended to study magnetic easy axis reorientation and the accompanying structure relaxation caused by the external magnetic field.

At high temperature, magnetite has its easy axis along cubic $\langle 111\rangle$, while it is [001] below $T_{\mathrm{V}}$. Since at $T_{\mathrm{V}}$ the structure changes from cubic to monoclinic, each of cubic $\langle 100\rangle$ may become an easy axis and the material breaks into several structural domains unless the external magnetic field $B>0.2 \mathrm{~T}$ along particular [001] is applied while cooling through the transition $[4,5]$; this particular direction would become the easy axis, which is also the monoclinic $c$ axis, doubled in comparison to

*corresponding author; e-mail: tarnawsk@uci.agh.edu.pl 
the cubic lattice constant. On the other hand, when a particular easy axis has been established and the magnetite sample is magnetized along another $\langle 100\rangle$ direction at temperatures slightly lower than $T_{\mathrm{V}}$, a reorientation of magnetic moments, i.e. axis switching (AS), may take place and this $\langle 100\rangle$ direction can, at least partly, become a new easy axis [5-7].

It is tempting to explain $\mathrm{AS}$ by $\mathrm{Fe}$ cations reorientation under the magnetic field to minimize their energy. This requires the simultaneous reorientation of magnetic and crystallographic axes, in particular. the monoclinic $c$ axis. Since the monoclinic cell is doubled in the $c$ direction, in comparison with cubic cell, the superstructure reflections, e.g. $\langle 8 \quad 81\rangle$ or $\langle 70-8\rangle$, which mark the onset of low temperature phase, should change their intensities when the external magnetic field is applied. Our experiment is aimed to check this conjecture.

\section{Experiment and discussion}

Measurements were performed on single crystals of magnetite skull melter grown from $99.99 \%$ pure $\mathrm{Fe}_{2} \mathrm{O}_{3}$ and annealed for stoichiometry. The results of magnetic AC susceptibility $\chi_{\mathrm{AC}}^{\prime}(T)$ show (see Fig. 1 ) the typical step at $T_{\mathrm{V}}(123.5 \mathrm{~K}$ ), proving that the annealing was effective. Magnetization was recorded on VSM setup (Lake Shore 7300) at magnetic field up to $1.0 \mathrm{~T}$ and at a few temperatures below $T_{\mathrm{V}}$. Crystal structure studies were done on a four-circle KM4 diffractometer with graphite-monochromatized Mo $K_{\alpha}$ radiation.
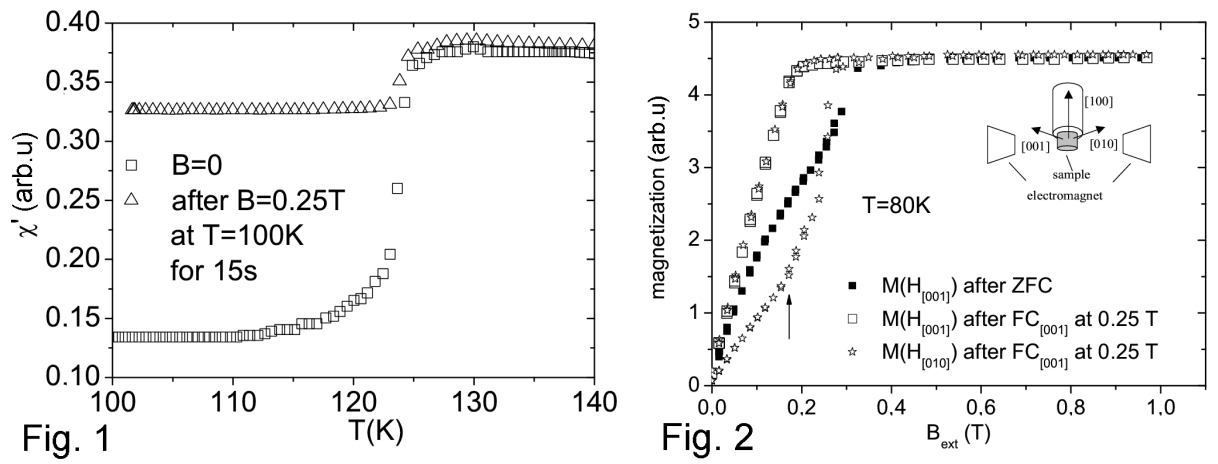

Fig. 1. $\chi_{\mathrm{AC}}^{\prime}(T)$ measured on heating without magnetic field treatment (open squares), and after the field shot at $100 \mathrm{~K}$. In both cases the characteristic step indicates the transition temperature.

Fig. 2. Magnetization vs. external magnetic field (e.g. $M\left(H_{[001]}\right)$ : magnetic field along [001], the field was first raised up to $1 \mathrm{~T}$ then lowered) after different field treatments: $\mathrm{ZFC}$ and $\mathrm{FC}_{[001]}$ along cubic [001]. The inset shows the experimental arrangement.

The experimental arrangement for magnetization studies is shown in the inset of Fig. 2. The results of zero field cooling (ZFC) and field cooling (FC) at $0.25 \mathrm{~T}$ along [001] are presented in Fig. 2; it is clear that although ZFC cannot 
define the easy magnetic axis, the cooling field of $0.25 \mathrm{~T}$ is sufficient to establish the easy axis along the required direction (open squares). After FC along [001], the sample was rotated and $M(H)$ was measured along [010] (an unspecified magnetic direction): the initial slope was completely different (proving FC has established the easy direction; stars) until it changed abruptly displaying the axis switching (marked by arrow). Let us note that on field lowering all three $M(H)$ curves are identical (shown only for the last experiment, stars, for clarity) and the same as that along [001] after FC.

This last observation strongly suggests that field treatment at $80 \mathrm{~K}$ can establish the easy magnetization direction equally well as FC from high temperatures. This is shown in Fig. 3, where we have collected the data from the last experiment from Fig. 2 (stars) and for ZFC sample that was, however, exposed to $B=1 \mathrm{~T}$ along [001] at $80 \mathrm{~K}$ (solid squares). Both samples were then rotated and measured along [010] direction, exhibiting nearly identical AS and the same $M(H)$ with field lowering. We have thus shown that the application of $1 \mathrm{~T}$ (in fact also $0.3 \mathrm{~T}$ and still lower field at higher $T$ ) in a low temperature phase is nearly as effective in establishing easy axis as field cooling at $0.25 \mathrm{~T}$.

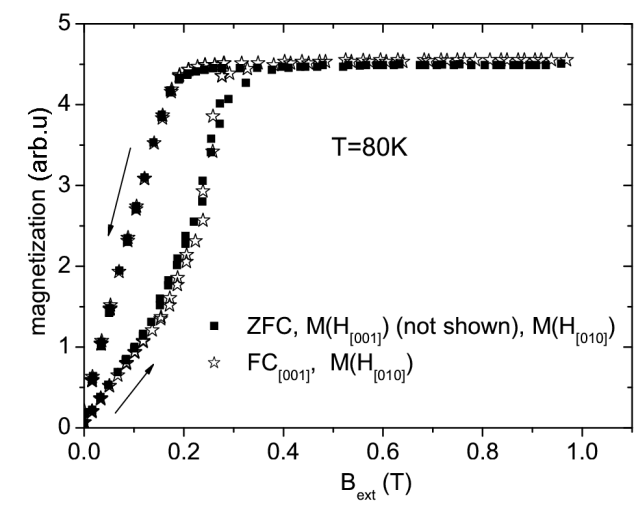

Fig. 3. Experimental proof that $\mathrm{FC}$ at $0.25 \mathrm{~T}$ along [001] is as effective in establishing easy axis as ZFC and subsequent field treatment along [001]: in both cases the axis switching along [010] takes place approximately at the same field (0.2 T). Arrows mark the field increasing or lowering.

It is now interesting to check if this relaxation of magnetic easy axis is accompanied by the simultaneous change of monoclinic $c$ axis. In other words, can the application of magnetic field change the intensity of superstructure peaks, related to the doubling of $c$ axis, i.e. of $\langle 881\rangle$ - or $\langle-807\rangle$-types?

The sample was first cooled down to $100 \mathrm{~K}$ and the integrated intensities of $\left(\begin{array}{lll}8 & 8 & 1\end{array}\right),\left(\begin{array}{lll}8 & 1 & 8\end{array}\right),\left(\begin{array}{lll}1 & 8 & 8\end{array}\right)$ and $\left(\begin{array}{lll}-8 & 0 & 7\end{array}\right),\left(\begin{array}{lll}0 & 7 & -8\end{array}\right),\left(\begin{array}{lll}7 & 0 & -8\end{array}\right)$, were measured. With the absence of magnetic field the intensities within each type of reflections should be comparable (see Fig. 4). Owing to nitrogen gas cooling system (Oxford 

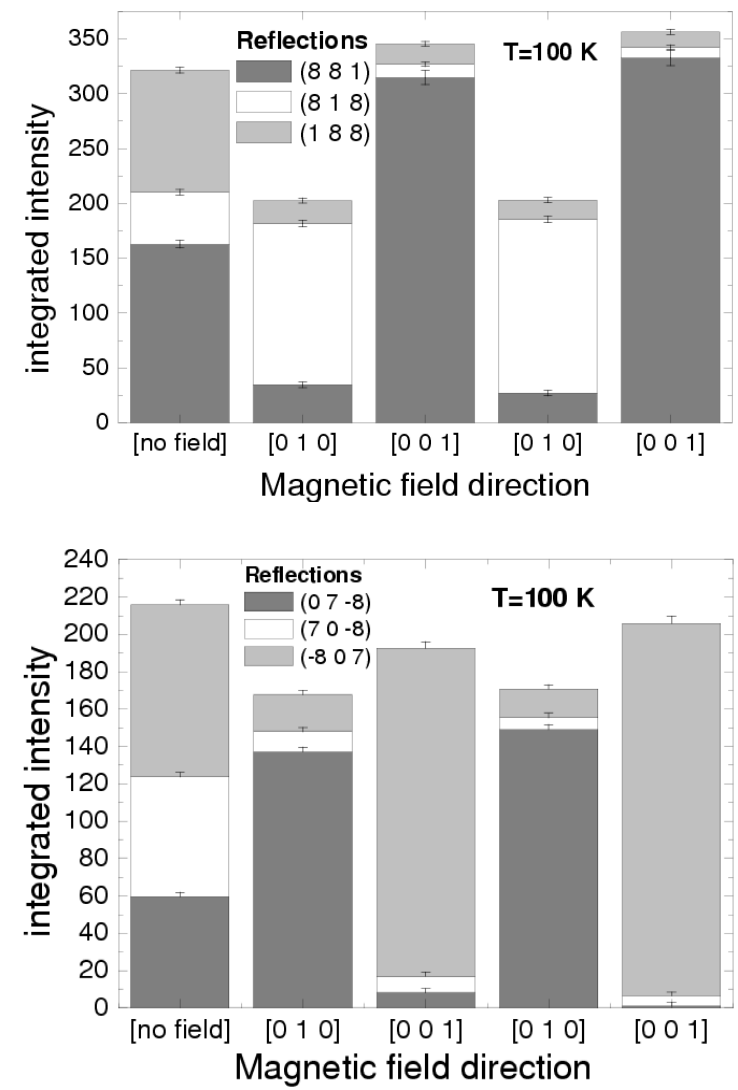

Fig. 4. Integrated intensities of $\langle 8 \quad 81\rangle$ and $\langle 07-8\rangle$ reflections measured at $100 \mathrm{~K}$ after ZFC (left bar) and after the field was applied in specified directions.

Cryosystems) we then managed to apply magnetic field (generated by the set of NdFeB magnets; $B \approx 0.3 \mathrm{~T}$ ) roughly in [010] and [001] directions (cubic indexing). Each time the experiment was repeated the integrated intensities from two sets of reflections were measured. The results are also shown in Fig. 4 and it is clear that the relative intensity changes in accordance with the field direction. We have thus shown that the application of magnetic field simultaneously rearranges the direction of easy magnetic axis and the $c$ monoclinic axis. The same conclusion may be drawn from another experiment, where magnetic $\chi_{\mathrm{AC}}$ is observed instead of structure: after a field shot the signal increases, proving the structure changes [8], see Fig. 1.

In conclusion, we have shown that the magnetic easy axis switching, which can be caused by the application of magnetic field, is accompanied by the relaxation of low temperature structure. 


\section{Acknowledgments}

This work is financially supported by the State Committee for Scientific Research.

\section{References}

[1] P. Novak, H. Stepankova, J. English, J. Kohout, V.A.M. Brabers, Phys. Rev B 61, 1256 (2000).

[2] G. Subias, J. Garcia, J. Blasco, M.G. Proietti, H. Renevier, M.C. Sanchez, Y. Joly, Phys. Rev. Lett. 93, 156408-1 (2004).

[3] J.P. Wright, J.P. Attfield, P.G. Radealli, Phys. Rev. B 66, 214422 (2002).

[4] Z. Kąkol, J.M. Honig, Phys. Rev. B 40, 9090 (1989).

[5] B.A. Calhoun, Phys. Rev. 94, 1577 (1954).

[6] E. Vittoratos, I. Baranov, P.P.M. Meincke, J. Appl. Phys. 42, 1633 (1971).

[7] Z. Ka̧kol, J. Solid State Chem. 88, 104 (1990).

[8] M. Bałanda, A. Wiecheć, D. Kim, Z. Kạkol, A. Kozłowski, P. Niedziela, J. Sabol, Z. Tarnawski, J.M. Honig, Eur. Phys. J. B 43, 201 (2005). 